Research Article

\title{
Complexity Induced by External Stimulations in a Neural Network System with Time Delay
}

\author{
Bin Zhen $\mathbb{D}^{1},{ }^{1}$ Dingyi Zhang, ${ }^{2}$ and Zigen Song ${ }^{3}{ }^{3}$ \\ ${ }^{1}$ School of Environment and Architecture, University of Shanghai for Science and Technology, Shanghai 200093, China \\ ${ }^{2}$ College of Food and Technology, Shanghai Ocean University, Shanghai 201306, China \\ ${ }^{3}$ College of Information Technology, Shanghai Ocean University, Shanghai 201306, China
}

Correspondence should be addressed to Zigen Song; zigensong@163.com

Received 8 July 2020; Accepted 12 August 2020; Published 7 September 2020

Academic Editor: Gonglin Yuan

Copyright (C) 2020 Bin Zhen et al. This is an open access article distributed under the Creative Commons Attribution License, which permits unrestricted use, distribution, and reproduction in any medium, provided the original work is properly cited.

Complexity and dynamical analysis in neural systems play an important role in the application of optimization problem and associative memory. In this paper, we establish a delayed neural system with external stimulations. The complex dynamical behaviors induced by external simulations are investigated employing theoretical analysis and numerical simulation. Firstly, we illustrate number of equilibria by the saddle-node bifurcation of nontrivial equilibria. It implies that the neural system has one/three equilibria for the external stimulation. Then, analyzing characteristic equation to find Hopf bifurcation, we obtain the equilibrium's stability and illustrate periodic activity induced by the external stimulations and time delay. The neural system exhibits a periodic activity with the increased delay. Further, the external stimulations can induce and suppress the periodic activity. The system dynamics can be transformed from quiescent state (i.e., the stable equilibrium) to periodic activity and then quiescent state with stimulation increasing. Finally, inspired by ubiquitous rhythm in living organisms, we introduce periodic stimulations with low frequency as rhythm activity from sensory organs and other regions. The neural system subjected by the periodic stimulations exhibits some interesting activities, such as periodic spiking, subthreshold oscillation, and bursting-like activity. Moreover, the subthreshold oscillation can switch its position with delay increasing. The neural system may employ time delay to realize Winner-Take-All functionality.

\section{Introduction}

Imitating the properties of a biological nerve system to build artificial neural network model plays an important role in the fields of neural network applications. Complexity and dynamical analysis in neural systems are important requirements for the application of optimization problem and associative memory. In fact, many neural network systems, such as Hopfield neural system, Cohen-Grossberg neural system, and cellular neural system, have been applied in many research fields such as associative memory, secure communication, and signal processing. Actually, associative memory storage in neural networks is defined by the stable equilibrium points, periodic orbits, and even chaotic attractors. Neural system receives external stimulations from sensory organs and brain regions and then produces different types of firing behaviors, such as periodic activity, spiking, bursting, and chaos to transfer and integrate neural information $[1,2]$. To reveal firing mechanism and study neural dynamics, many neural system models have been constructed. The evolution of system is governed by the assumed dynamics of units and their interactions [3]. Time delay is an inevitable factor in signal transmission due to the finite propagation velocity and switching speed. Recently, delayed neural systems with few units have been proposed to get a deep understanding of the neural dynamics [4-6].

In this paper, we investigate a simplified delay neural system with external stimulations. The Wilson-Cowan (W-C) neural model with time delay [7] is chosen to describe dynamical behavior of individual neurons or neural function units. The $\mathrm{W}-\mathrm{C}$ model is a set of differential equations that represent time evolution of neural units, using sigmoidal function to describe neural interaction. The W-C neural system has been used to address many problems in 
computational neuroscience [8]. The mathematical characteristics of single and two-coupling W-C neural system have been studied extensively because of mathematical tractability and rich dynamical properties [9, 10]. For example, Borisyuk and Kirillov [11] found a coexistence region with equilibrium and periodic solution in $\mathrm{W}-\mathrm{C}$ model. The dynamics and bifurcations of two coupled $\mathrm{W}-\mathrm{C}$ oscillators with four different connection types were investigated [12]. Maruyama et al. [13] elucidated the chaos mechanism in coupled W-C oscillators. Further, the modified W-C oscillator with delayed self-connection was proposed by Monteiro et al. [14]. Song and Xu have focused on the effects of external inputs in delayed $\mathrm{W}-\mathrm{C}$ oscillator $[15,16]$. Two coupled W-C models exhibited a chaotic oscillation forced by periodic input [13]. Moreover, the neural system had coexistence of oscillatory and steady states. The periodic stimulation transformed the level of activity to high or low $[17,18]$. However, to the best of our knowledge, there is no published report on dynamical behavior of $\mathrm{W}-\mathrm{C}$ neural model with time delay and external stimulations. This is our motivation of the present research.

The $\mathrm{W}-\mathrm{C}$ neural system with time delay and external stimulations is described by the following delayed differential equation:

$$
\left\{\begin{array}{l}
\dot{u}_{1}(t)=-u_{1}(t)+f\left(c_{1} u_{1}(t)+c_{2} u_{2}(t-\tau)+I_{1}\right), \\
\dot{u}_{2}(t)=-u_{2}(t)+f\left(c_{3} u_{2}(t)+c_{4} u_{1}(t-\tau)+I_{2}\right)
\end{array}\right.
$$

where $u_{1}(t)$ and $u_{2}(t)$ denote neural activities at time $t, c_{i}$ $(i=1, \ldots, 4)$ represent synaptic weights for inhibition $\left(c_{i}<0\right)$ and excitation $\left(c_{i}>0\right), \tau$ is a transmission delay, and $I_{1}$ and $I_{2}$ are external stimulations, $f(u)$ is neural activation function and chosen as $f(u)=1 /\left(1+e^{-u}\right)$ in this paper.

The outline of this paper is as follows: in Section 2, we illustrate number of equilibria by saddle-node bifurcation of nontrivial equilibria. The system exhibits one/three equilibria for different values of external stimulations. In Section 3 , we analyze the equilibrium's stability and demonstrate a periodic activity, which can be induced by both external stimulations and time delay. The neural system exhibits a periodic activity with delay increasing. Further, the external stimulations can induce and suppress the periodic activity. The system dynamics can be transformed from quiescent state to periodic activity and then to quiescent state with increase of the external stimulations. In Section 4, we introduce periodic stimulations as rhythm activity from sensory organs and another region. The neural system subjected by the periodic stimulations exhibits periodic spiking, subthreshold, and bursting-like activities. Conclusions are given in Section 5.

\section{Saddle-Node Bifurcation of Nontrivial Equilibrium}

We start with equilibrium analysis by employing the dynamical bifurcation theory. It is obvious that system (1) just has nontrivial equilibrium labeled as $\left(u_{10}, u_{20}\right)$ because the neural activation function $f(u)=1 /\left(1+e^{-u}\right)$ is not an origin symmetry. By letting $\dot{u}_{1}=0$ and $\dot{u}_{2}=0$ in system (1), we have the nontrivial equilibrium $\left(u_{10}, u_{20}\right)$ satisfied with

$$
\left\{\begin{array}{l}
u_{10}=f\left(c_{1} u_{10}+c_{2} u_{20}+I_{1}\right), \\
u_{20}=f\left(c_{3} u_{20}+c_{4} u_{10}+I_{2}\right) .
\end{array}\right.
$$

The number and value of the nontrivial equilibrium just depend on synaptic weights $c_{i},(i=1, \ldots, 4)$ and external stimulations $I_{1}$ and $I_{2}$. Since time delay has no impact on the number and value of system equilibria, we have rewritten system (1) as the following nondelayed system, which is

$$
\left\{\begin{array}{l}
\dot{u}_{1}(t)=-u_{1}(t)+f\left(c_{1} u_{1}(t)+c_{2} u_{2}(t)+I_{1}\right), \\
\dot{u}_{2}(t)=-u_{2}(t)+f\left(c_{3} u_{2}(t)+c_{4} u_{1}(t)+I_{2}\right) .
\end{array}\right.
$$

By $x_{1}(t)=u_{1}(t)-u_{10}, x_{2}(t)=u_{2}(t)-u_{20}$, we obtain the corresponding linearization system:

$$
\left\{\begin{array}{l}
\dot{x}_{1}(t)=-x_{1}(t)+c_{1} M x_{1}(t)+c_{2} M x_{2}(t), \\
\dot{x}_{2}(t)=-x_{2}(t)+c_{3} N x_{2}(t)+c_{4} N x_{1}(t),
\end{array}\right.
$$

where

$$
\begin{gathered}
M=\frac{e^{-m_{0}}}{\left(1+e^{-m_{0}}\right)^{2}}>0, \\
N=\frac{e^{-n_{0}}}{\left(1+e^{-n_{0}}\right)^{2}}>0, \\
m_{0}=c_{1} u_{10}+c_{2} u_{20}+I_{1}, \\
n_{0}=c_{3} u_{20}+c_{4} u_{10}+I_{2} .
\end{gathered}
$$

The corresponding characteristic equation is

$$
\left|\begin{array}{cc}
\lambda+1-c_{1} M & -c_{2} M \\
-c_{4} N & \lambda+1-c_{3} N
\end{array}\right|=0,
$$

which is

$$
\begin{aligned}
\lambda^{2} & +\left(-c_{1} M-c_{3} N+2\right) \lambda+\left(c_{1} c_{3}-c_{2} c_{4}\right) M N-c_{3} N \\
& -c_{1} M+1=0 .
\end{aligned}
$$

In fact, the critical value of equilibrium's number corresponds to a static bifurcation $[19,20]$. So, in Section 3, we will describe the change of the number of equilibria by bifurcation analysis. It follows from bifurcation theory that system (1) has a static bifurcation at the nontrivial equilibrium $\left(u_{10}, u_{20}\right)$ if the following equation is valid, which is

$$
\left(c_{1} c_{3}-c_{2} c_{4}\right) M N-c_{3} N-c_{1} M+1=0 .
$$

It should be noted that $M$ and $N$ entirely depend on synaptic weights $c_{i},(i=1, \ldots, 4)$ and external stimulations $I_{1}$ and $I_{2}$. So, equation (8) is a transcendental and complicated equation. The static bifurcation point cannot be illustrated in theoretical expressions. But, for the fixed system parameters, the static bifurcation points can be obtained by numerical computation. Further, we choose system parameters as $c_{1}=-3, c_{2}=-10, c_{3}=-3$, and $c_{4}=-10$. For different values of the external stimulations $I_{1}$ and $I_{2}$, we can 
show dynamic nullclines to demonstrate the types of bifurcation of nontrivial equilibrium, which is

$$
\left\{\begin{array}{l}
I_{1}=3 u_{10}+10 u_{20}-\ln \left(\frac{1}{u_{10}-1}\right) \\
I_{2}=3 u_{20}+10 u_{10}+\ln \left(\frac{1}{u_{20}-1}\right) .
\end{array}\right.
$$

The number of equilibria can be obtained by dynamic nullclines, as shown in Figure 1. It follows from Figure 1(a) that the dynamic nullclines just have one intersection point for $I_{1}=8$ and $I_{2}=5$, which implies system (1) has just one nontrivial equilibrium. However, when external stimulations $I_{1}$ and $I_{2}$ are fixed as $I_{1}=8, I_{2}=7$, the dynamic nullclines have three intersection points with a nonsymmetrical pattern, as shown in Figure 1(b). It implies that system (1) exhibits a saddle-node bifurcation of nontrivial equilibrium. Two new nontrivial equilibria generate with external stimulations increasing. System (1) exhibits three equilibria. Similar results are obtained for system (1), which is one equilibrium for $I_{1}=2$ and $I_{2}=-2$ (Figure $1(\mathrm{c})$ ) and three equilibria for $I_{1}=2$ and $I_{2}=2$ (Figure $1(\mathrm{~d})$ ).

\section{Stability Analysis and Period Activity}

In this section, we will analyze the equilibrium's stability and find periodic activity induced by both external stimulations and time delay. To this end, using $x_{1}=u_{1}-u_{10}$ and $x_{2}=u_{2}-u_{20}$, we firstly obtain a linearizing system at the equilibrium $\left(u_{10}, u_{20}\right)$, which is

$$
\left\{\begin{array}{l}
\dot{x}_{1}(t)=-x_{1}(t)+c_{1} M x_{1}(t)+c_{2} M x_{2}(t-\tau) \\
\dot{x}_{2}(t)=-x_{2}(t)+c_{3} N x_{2}(t)+c_{4} N x_{1}(t-\tau)
\end{array}\right.
$$

The characteristic equation of system (10) is

$$
\begin{aligned}
\lambda^{2} & +\left(-c_{1} M-c_{3} N+2\right) \lambda-c_{2} c_{4} M N e^{-2 \lambda \tau}+c_{1} c_{3} M N \\
& -c_{3} N-c_{1} M+1=0 .
\end{aligned}
$$

It follows that the equilibrium of system (1) is locally stable when all eigenvalues of equation (11) present negative real parts. Supposing $\tau=0$ in equation (11) produces

$$
\lambda^{2}+\left(-c_{1} M-c_{3} N+2\right) \lambda+\left(c_{1} c_{3}-c_{2} c_{4}\right) M N-c_{3} N-c_{1} M+1=0 .
$$

Using the Routh-Hurwitz criterion, we obtain a necessary and sufficient condition to assure the equilibrium of system (1) has local stability, which is

$$
\left\{\begin{array}{l}
c_{1} M+c_{3} N<2, \\
c_{1} M+c_{3} N-c_{1} c_{3} M N+c_{2} c_{4} M N<1 .
\end{array}\right.
$$

With delay $\tau$ increasing, the system equilibrium may lose its stability and evolve into a periodic activity. To obtain the critical values, supposing $\lambda=i \omega(\omega>0)$ is a pure imaginary root of the characteristic equation (11), we have

$$
\begin{aligned}
\omega^{2} & +\left(-c_{1} M-c_{3} N+2\right) i \omega-c_{2} c_{4} M N e^{-2 i \omega \tau}+c_{1} c_{3} M N \\
& -c_{3} N-c_{1} M+1=0 .
\end{aligned}
$$
yields

Separating equation (14) into real and imaginary parts $\left\{\begin{array}{l}1-c_{1} M-c_{3} N+c_{1} c_{3} M N-\omega^{2}-c_{2} c_{4} M N \cos (2 \omega \tau)=0, \\ 2 \omega-c_{1} M \omega-c_{3} N \omega+c_{2} c_{4} M N \sin (2 \omega \tau)=0 .\end{array}\right.$

Eliminating $\tau$ from equation (15), one has

$$
\left\{\begin{array}{l}
\cos (2 \omega \tau)=\frac{1-c_{1} M-c_{3} N+c_{1} c_{3} M N-\omega^{2}}{c_{2} c_{4} M N}, \\
\sin (2 \omega \tau)=-\frac{2 \omega-c_{1} M \omega-c_{3} N \omega}{c_{2} c_{4} M N}
\end{array}\right.
$$

By $\cos ^{2}(2 \omega \tau)+\sin ^{2}(2 \omega \tau)=1$, we have

$$
L(\omega)=\omega^{4}+p_{0} \omega^{2}+q_{0}=0,
$$

where

$$
\left\{\begin{array}{l}
p_{0}=2-2 c_{1} M-2 c_{3} N+c_{1}^{2} M^{2}+c_{3}^{2} N^{2}, \\
q_{0}=\left(1-c_{1} M-c_{3} N+c_{1} c_{3} M N\right)^{2}-\left(c_{2} c_{4} M N\right)^{2} .
\end{array}\right.
$$

In general, based on conditions $p_{0}<0, q_{0}>0$, and $p_{0}^{2}-4 q_{0}>0$, equation (17) may have at most two positive roots $\omega_{i}, i=1,2$, which are the frequencies of the periodic activity

$$
\omega_{1,2}=\sqrt{\frac{-p_{0} \pm \sqrt{p_{0}^{2}-4 q_{0}}}{2}} .
$$

Then, equation (14) has the critical delayed values of the Hopf bifurcation, that is

$$
\tau_{i}^{j}=\frac{\phi_{i}+2 j \pi}{\omega_{i}}, \quad i=1,2 ; j=0,1,2, \ldots,
$$

where $\phi_{i} \in[0,2 \pi)$ and satisfies with

$$
\left\{\begin{array}{l}
1-c_{1} M-c_{3} N+c_{1} c_{3} M N-\omega_{i}^{2}-c_{2} c_{4} M N \cos \phi_{i}=0, \\
2 \omega_{i}-c_{1} M \omega_{i}-c_{3} N \omega_{i}+c_{2} c_{4} M N \sin \phi_{i}=0 .
\end{array}\right.
$$

Define

$$
\tau^{0}=\min \left\{\tau_{i}^{0}: i=1,2 .\right\} .
$$

The Hopf bifurcation happens when the system eigenvalues cross the imaginary axis with nonzero velocity. Differentiating $\lambda$ with $\tau$ in (11), one has the crossing velocity:

$$
\lambda^{\prime}(\tau)=\frac{2 c_{2} c_{4} M N \lambda}{e^{2 \lambda \tau}\left(c_{1} M+c_{3} N-2 \lambda-2\right)-2 c_{2} c_{4} M N \tau} .
$$




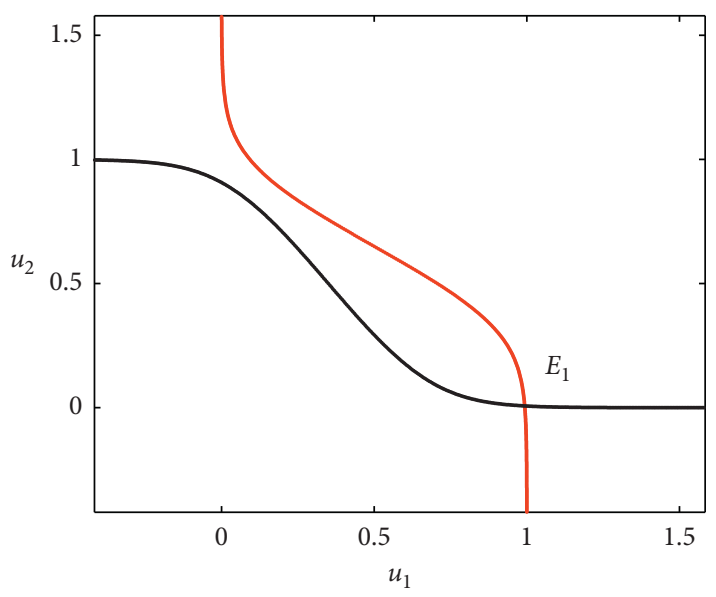

(a)

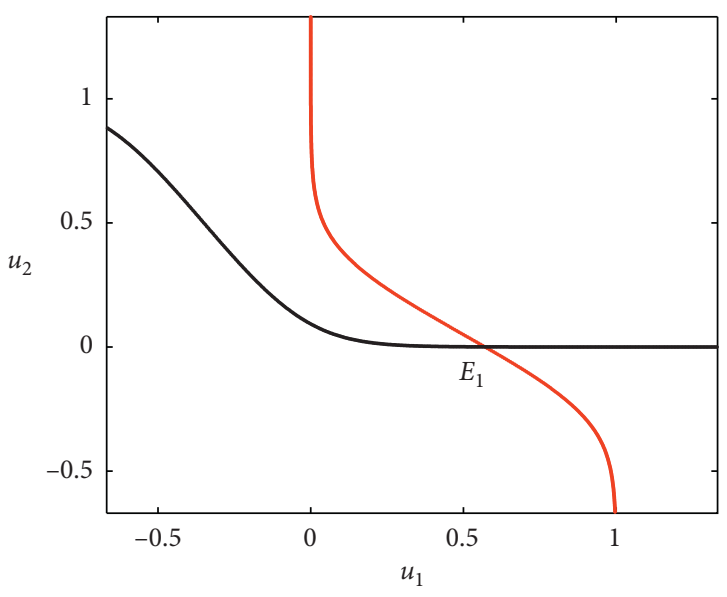

(c)

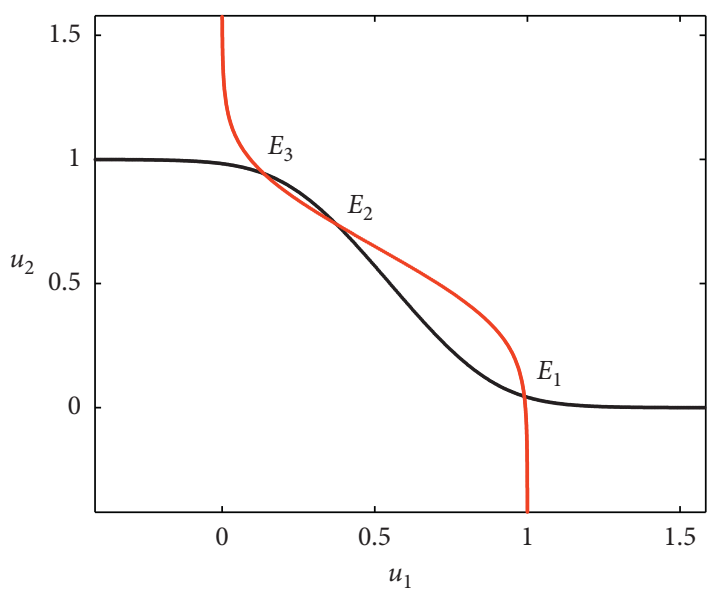

(b)

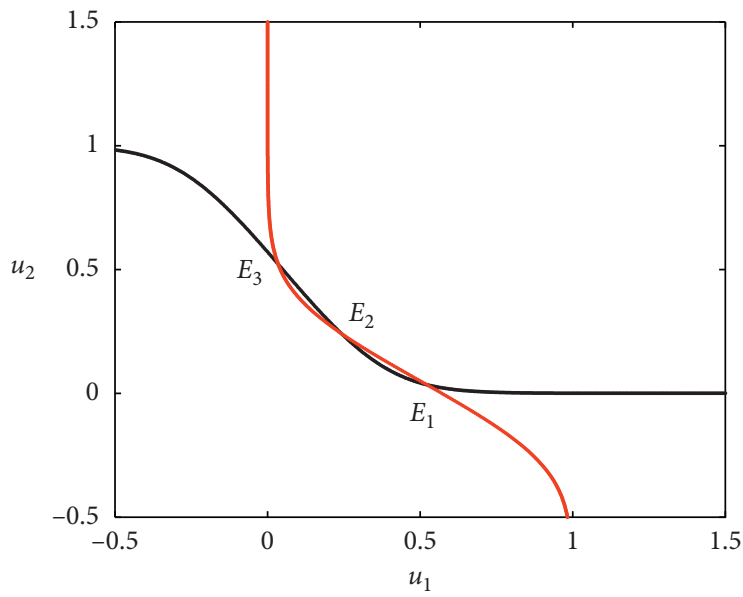

(d)

FIGURE 1: Intersection points of dynamic nullclines illustrate the saddle-node bifurcation of nontrivial equilibrium, where (a) one nontrivial equilibrium for $I_{1}=8$ and $I_{2}=5$, (b) three equilibria for $I_{1}=8$ and $I_{2}=7$, (c) one equilibrium for $I_{1}=2$ and $I_{2}=-2$, and (d) three equilibria for $I_{1}=2$ and $I_{2}=2$, where the other parameters are $c_{1}=-3, c_{2}=-10, c_{3}=-3$, and $c_{4}=-10$.

By the Hopf bifurcation theory, we obtain the following conclusion with condition (11). If $L(\omega)=0$ has at most two positive roots $\omega_{i}, i=1,2$, there is a critical value of delay $\tau^{0}$ defined by (22). All eigenvalues have negative real parts for $\tau \in\left(0, \tau^{0}\right)$, where the dynamics of system (1) is asymptotically stable. Furthermore, system (1) has a Hopf bifurcation with the critical delayed value $\tau=\tau^{0}$ when $\operatorname{Re}\left(\lambda_{\prime}(\tau)\right) \neq 0$. It implies that the system has a periodic activity near the equilibrium $\left(u_{10}, u_{20}\right)$ for $\tau \in\left(\tau^{0},+\infty\right)$.

For example, we choose system parameters as $c_{1}=-3$, $c_{2}=-10, c_{3}=3, c_{4}=5, I_{1}=2$, and $I_{2}=-2$. It follows that $L(\omega)=0$ has a positive $\operatorname{root} \omega=0.6133$. The minimum delay of the Hopf bifurcation is $\tau^{0}=1.2422$ by equation (18). All eigenvalues of equation (11) have negative real parts for the delayed interval $\tau \in(0,1.2422)$. At this time, the system equilibrium is locally stable. It follows from Figure 2(a) that the maximum real part of eigenvalues is $\operatorname{Re}(\lambda)=-0.0389$ for the fixed delay $\tau=1$. The corresponding time history is illustrated in Figure 3(a). The trajectory evolves into the nontrivial equilibrium. Furthermore, system (1) undergoes a Hopf bifurcation when $\tau$ passes through the critical delayed value $\tau^{0}=1.2422$. The maximum real part of eigenvalues will change its sign from negative to positive. At least a root of equation (9) has a positive part for $\tau \in(1.2422, \infty)$, as shown in Figure 2(b) for the fixed delay $\tau=2$. The system dynamic will lose its stability and enter a stable periodic activity, as shown in Figure 3(b). The real parts of system eigenvalues with $\tau$ increasing are shown in Figure 4 .

On the other hand, external stimulations can induce and suppress the periodic activity in system (1). With increasing of the external stimulations, the system dynamics can switch from quiescent state to periodic activity and then enter into the quiescent state. For example, we increase external stimulation $I_{2}$ and fix other parameters as $c_{1}=-3, c_{2}=-10$, $c_{3}=-3, \quad c_{4}=-10, I_{1}=2$, and $\tau=2$. It follows from Figure 5(a) that the system trajectories evolve into a nontrivial equilibrium for $I_{2}=0$. The equilibrium of system is locally stable. With increasing of external stimulation to $I_{2}=2$, the system dynamic loses its stability and enters into a stable periodic activity, as shown in Figure 5(b). It implies that the external stimulation induces a periodic activity in system (1). Further, the intensity of periodic activity is 


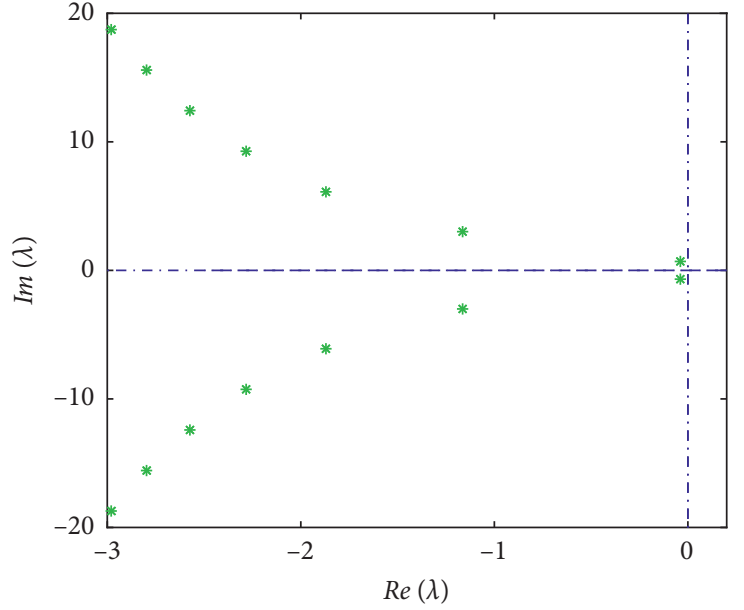

(a)

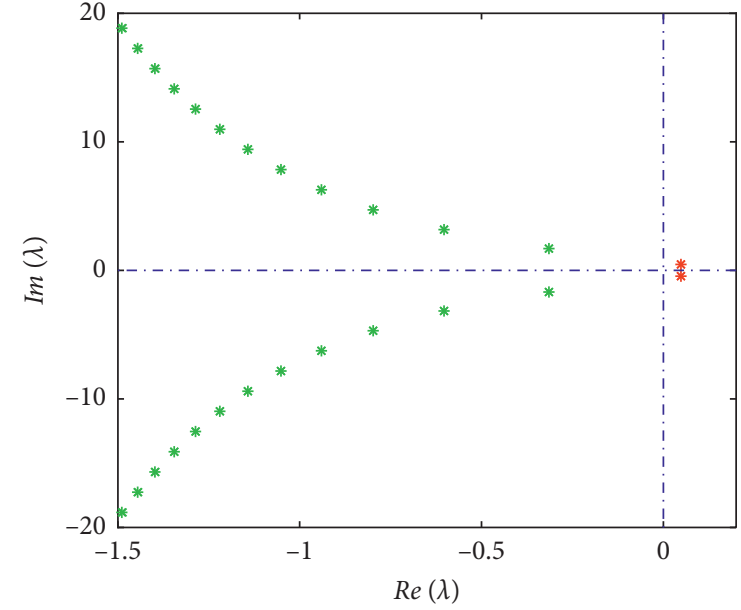

(b)

Figure 2: Partial eigenvalues of the system equilibrium $\left(u_{10}, u_{20}\right)$ with time delay $\tau$ increasing (a) $\tau=1$ and (b) $\tau=2$ for the fixed parameters $c_{1}=-3, c_{2}=-10, c_{3}=3, c_{4}=5, I_{1}=2$, and $I_{2}=-2$.

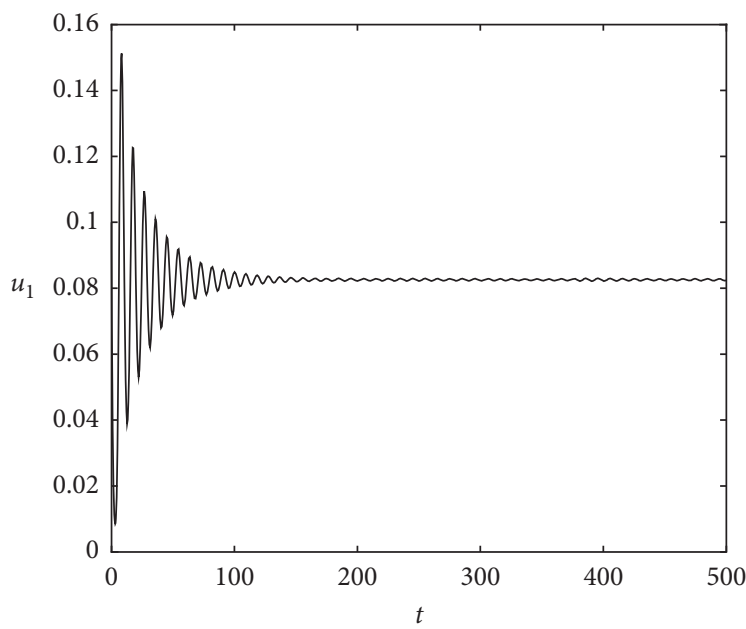

(a)

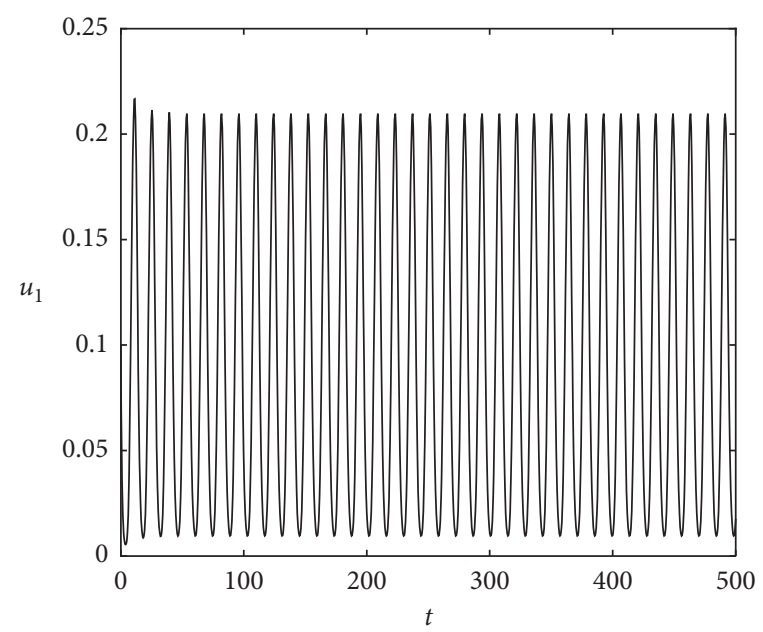

(b)

Figure 3: Time histories of system (1) with the delay (a) $\tau=1$ and (b) $\tau=2$ for the fixed parameters $c_{1}=-3, c_{2}=-10, c_{3}=3, c_{4}=5, I_{1}=2$, and $I_{2}=-2$.

enhanced by external stimulation $I_{2}$. It follows from Figure $5(\mathrm{c})$ that the amplitude can reach to $u_{1}=0.5$ for external stimulation $I_{2}=6$. However, when external stimulation is increased into $I_{2}=8$, the periodic activity evolves into a quiescent state, as shown in Figure 5(d). The system trajectories enter a nontrivial equilibrium again. The system equilibrium regains its stability. The external stimulations suppress the periodic activity in system (1).

\section{Complex Activity Excited by Periodic Stimulations}

In the section above, we have studied the equilibrium stability and find a periodic activity, where system (1) has external stimulations $I_{1}$ and $I_{2}$, which are constant values. In fact, rhythm stimulation is ubiquitous in living organisms.
So, in this section, we will analyze dynamical activity of the neural system encountered external stimulation with periodical rhythm. The periodic stimulations with low frequency are introduced as rhythm activity from sensory organs and other regions. The neural system considered in this section is described by the following differential equations:

$$
\left\{\begin{array}{l}
\dot{u}_{1}(t)=-u_{1}(t)+f\left(c_{1} u_{1}(t)+c_{2} u_{2}(t-\tau)+\left(I_{1}+A_{1} \cos (v t)\right)\right), \\
\dot{u}_{2}(t)=-u_{2}(t)+f\left(c_{3} u_{2}(t)+c_{4} u_{1}(t-\tau)+\left(I_{2}+A_{2} \sin (v t)\right)\right),
\end{array}\right.
$$

where $A_{1}$ and $A_{2}$ are amplitudes of external stimulations with periodical rhythm and $v$ is frequency. Physically, in some experimental studies of living organisms, periodic stimulations are used by injecting sinusoidal current into neurons, such as the squid giant axons [21], snail neurons [22], and lobster CPGs [23]. Some complex activity of 


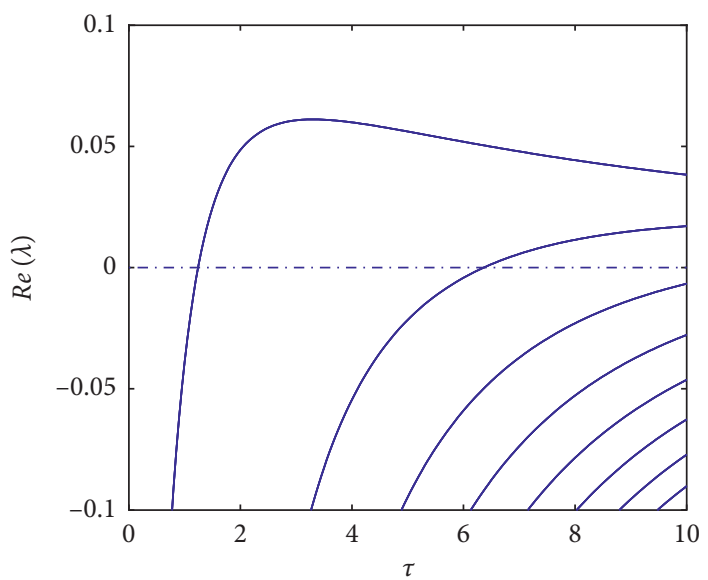

Figure 4: The real parts of system eigenvalues with $\tau$ increasing for the fixed parameters $c_{1}=-3, c_{2}=-10, c_{3}=3, c_{4}=5, I_{1}=2$, and $I_{2}=-2$.

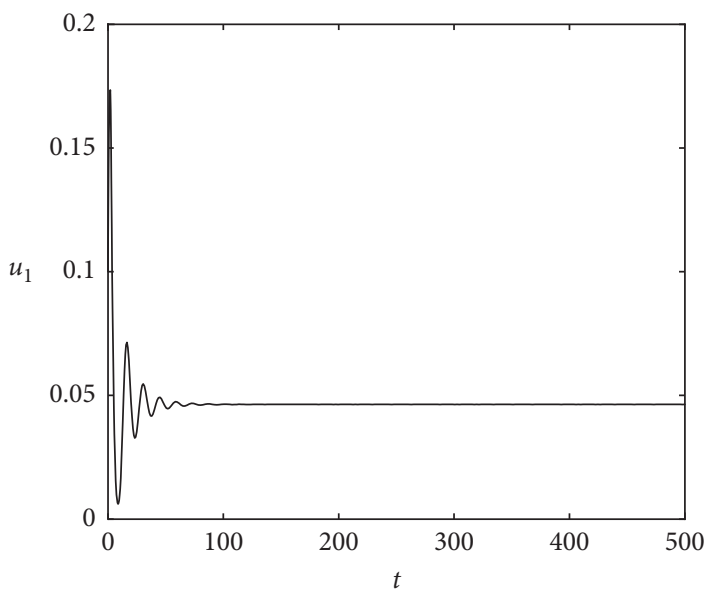

(a)

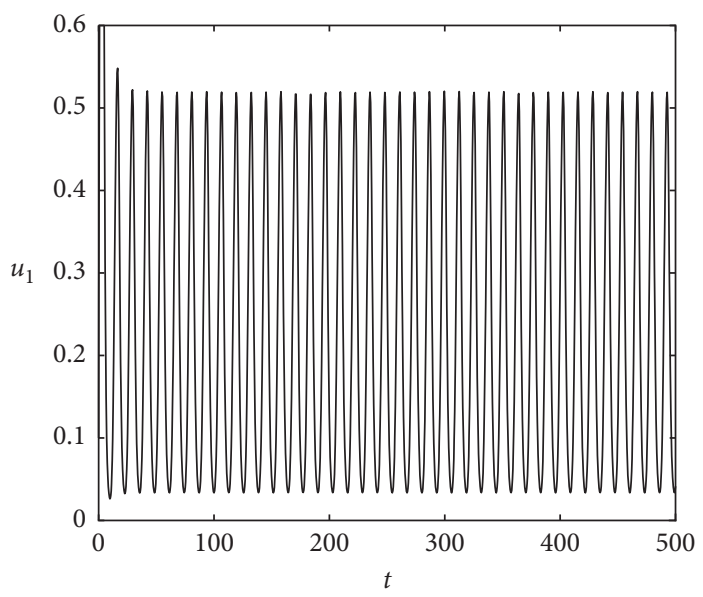

(c)

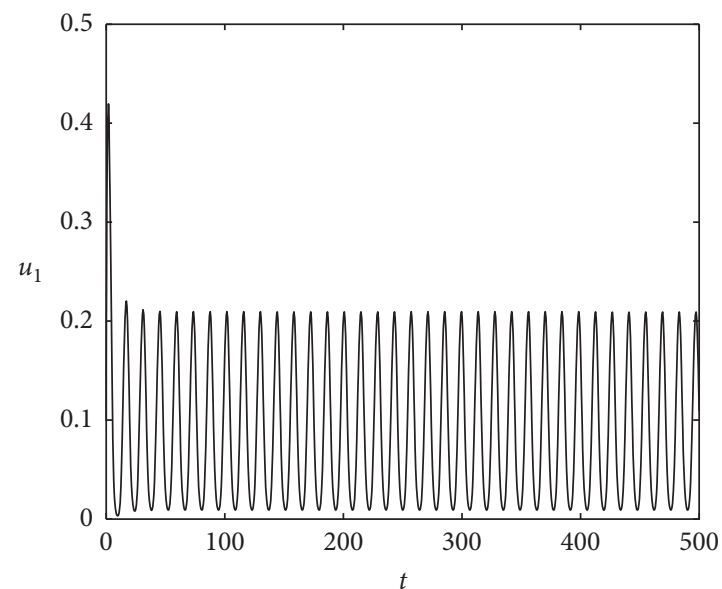

(b)

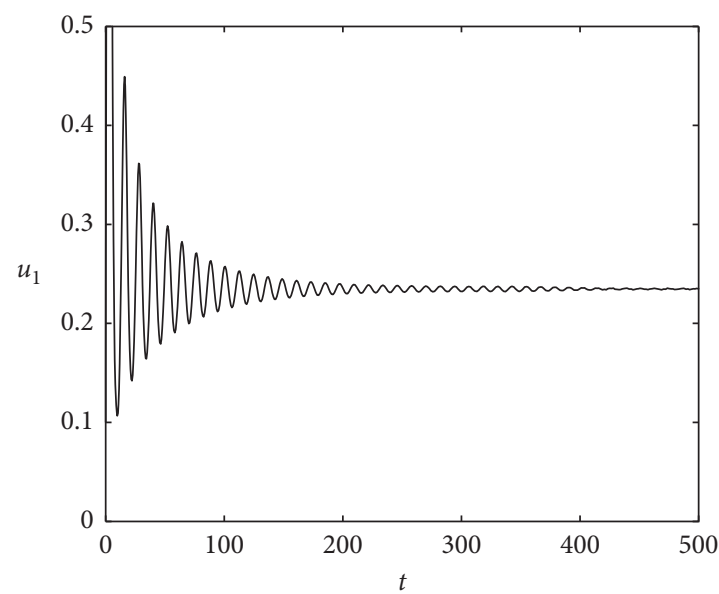

(d)

Figure 5: Time histories of system (1) with external stimulation increasing (a) $I_{2}=0$, (b) $I_{2}=2$, (c) $I_{2}=6$, and (d) $I_{2}=8$ for the fixed parameters $c_{1}=-3, c_{2}=-10, c_{3}=-3, c_{4}=-10, I_{1}=2$, and $\tau=2$. 


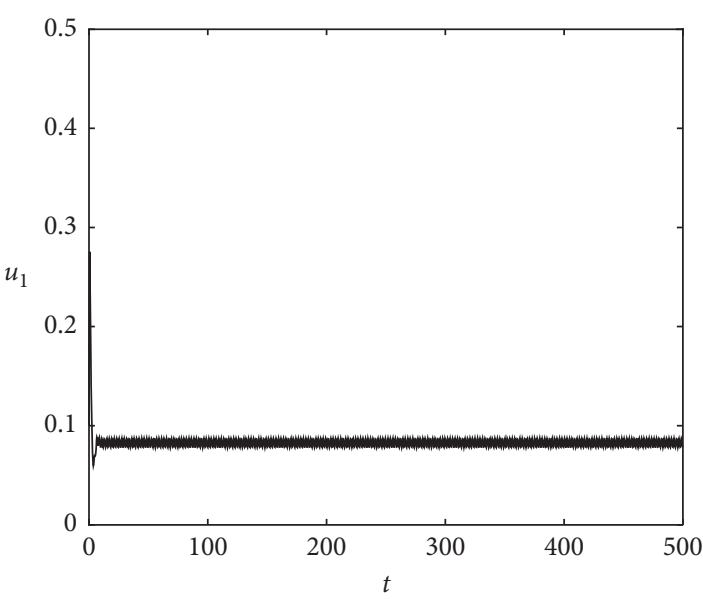

(a)

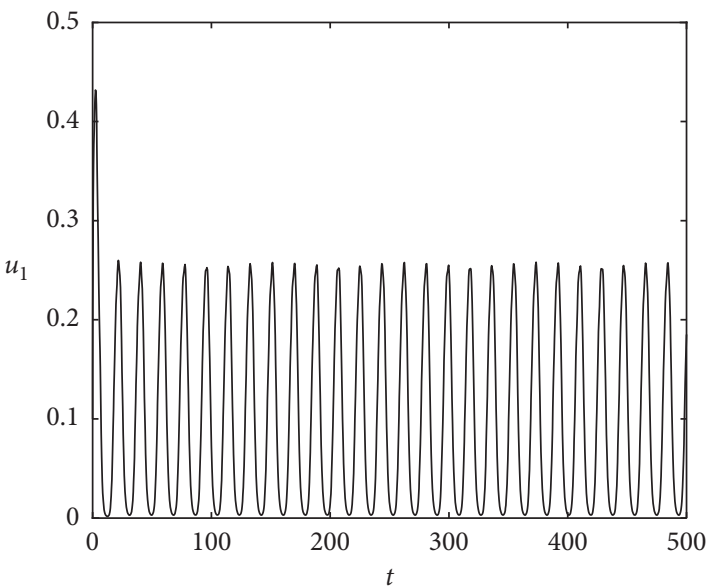

(c)

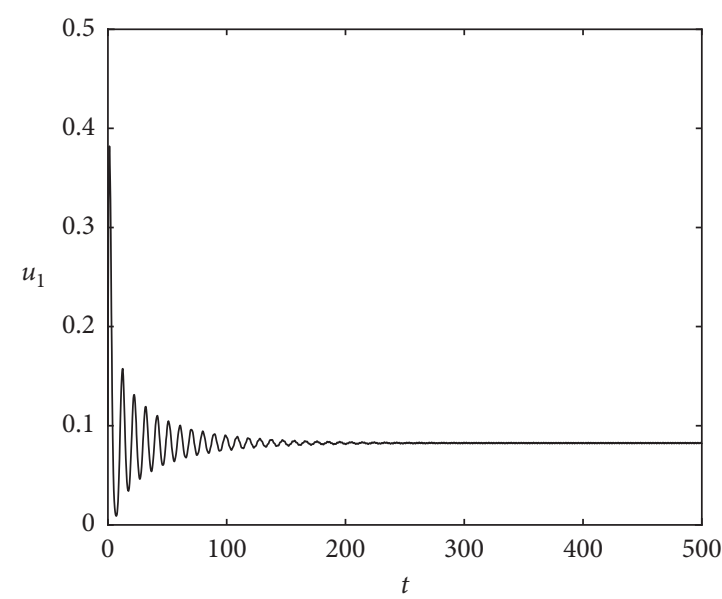

(b)

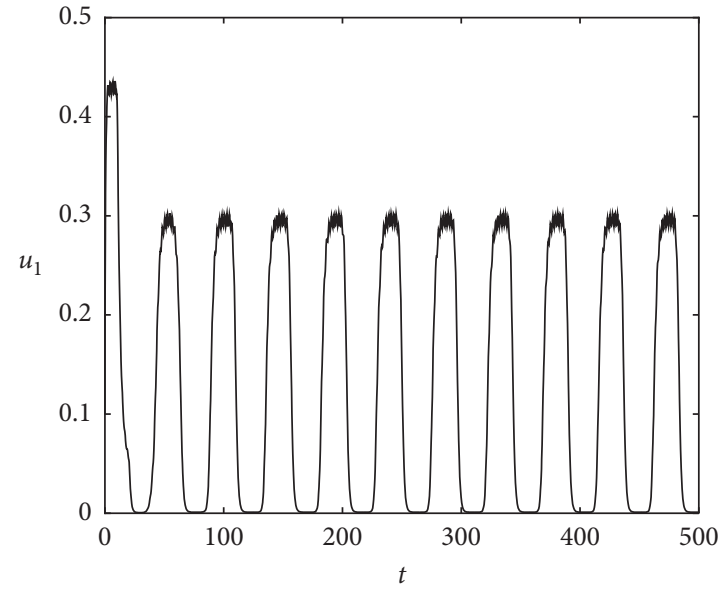

(d)

Figure 6: Time histories of system (24) with delay increasing (a) $\tau=0.1$, (b) $\tau=1$, (c) $\tau=3$, and (d) $\tau=10$ for the fixed parameters $c_{1}=-3$, $c_{2}=-10, c_{3}=3, c_{4}=5, I_{1}=2$, and $I_{2}=-2$.

neurons, such as periodic spiking, subthreshold, bursting, and even chaos behaviors are illustrated for external stimulations with periodical rhythm.

It follows from equilibrium analysis in Section 2 that neural system (1) exhibits one equilibrium and three equilibria for different parameter values. Further, with time delay and external stimulations, the neural system illustrates a periodic activity from a quiescent state and even regains the quiescent state. So, in Section 5, we exhibit dynamic activity of neural system (24) with two cases. One is $c_{1}=-3$, $c_{2}=-10, c_{3}=3, c_{4}=5, I_{1}=2$, and $I_{2}=-2$ for system (1) having one equilibrium, and the other is $c_{1}=-3, c_{2}=-10$, $c_{3}=-3, c_{4}=-10, I_{1}=8$, and $I_{2}=7$ for three equilibria. Further, by choosing $A_{1}=0.1, A_{2}=0.1$, and $v=3$ as the periodic stimulations, we illustrate the effect of time delay and external stimulation on system activity. The results show that the neural system subjected by periodic stimulations exhibits some interesting activities, such as periodic spiking, subthreshold oscillation, and bursting-like activity.

Firstly, we choose system parameters as $c_{1}=-3$, $c_{2}=-10, c_{3}=3, c_{4}=5, I_{1}=2$, and $I_{2}=-2$, where system (1) has one equilibrium. The neural activities of neural system (24) subjected by periodic stimulations are illustrated in Figure 6 for the different values of time delay. It follows from Figure 6(a) that system (24) exhibits a subthreshold oscillation for small delay $\tau=0.1$. Further, the amplitude of subthreshold oscillation decreases with time delay increasing. The subthreshold oscillation degenerates into a quiescent state, as shown in Figure 6(b) for $\tau=1$. On the other hand, with delay further increasing, the quiescent state can be excited, and the subthreshold oscillation enters into a periodic spiking, as shown in Figure 6(c) for $\tau=3$. At last, when time delay is chosen as $\tau=10$, the periodic spiking transforms into a bursting-like activity, as shown in Figure 6(d).

For case 2, system parameters are fixed as $c_{1}=-3$, $c_{2}=-10, c_{3}=-3, c_{4}=-10, I_{1}=8$, and $I_{2}=8$, where neural system (1) exhibits three equilibria by saddle-node bifurcation of nontrivial equilibrium. The activities of neural system (24) subjected by periodic stimulations illustrate the top/down subthreshold oscillation and periodic spiking, as shown in Figure 7. It follows from Figure 7(a) that the neural system (24) exhibits a top subthreshold oscillation because of the top equilibrium in system (1) for delay $\tau=1.5$. With 


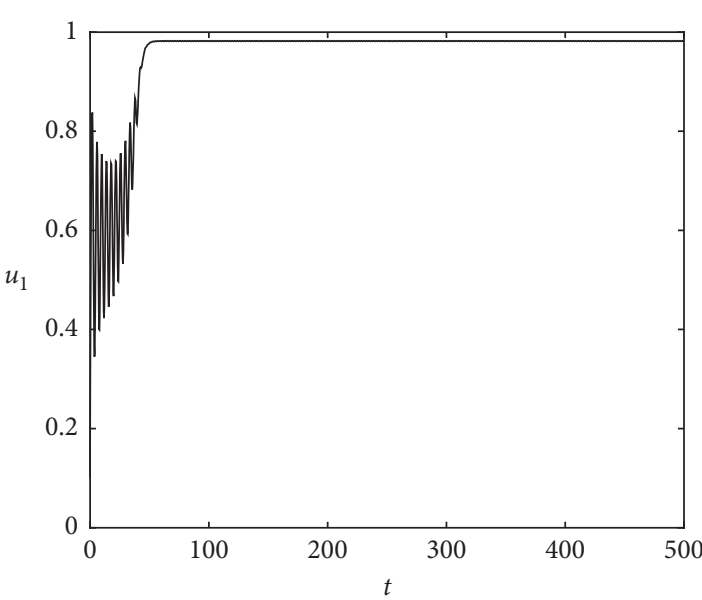

(a)

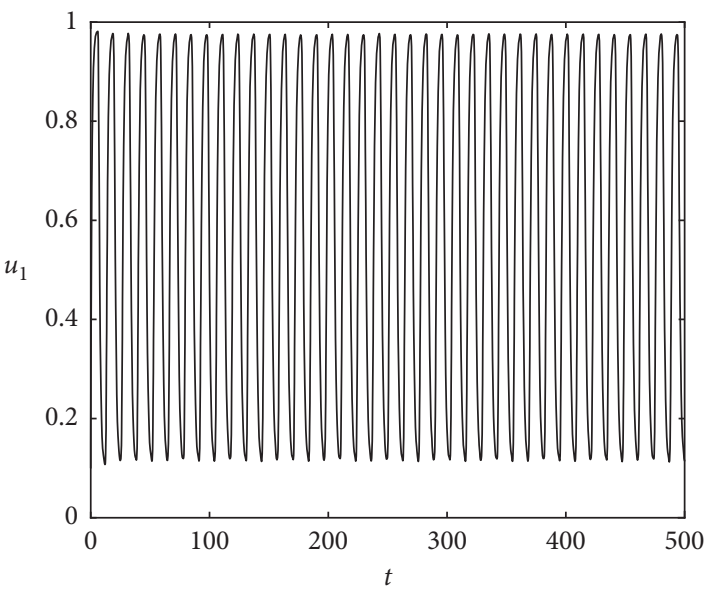

(c)

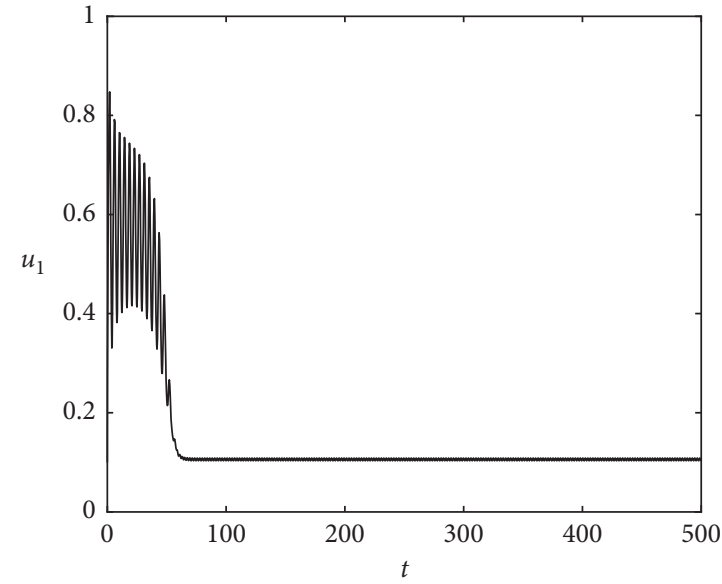

(b)

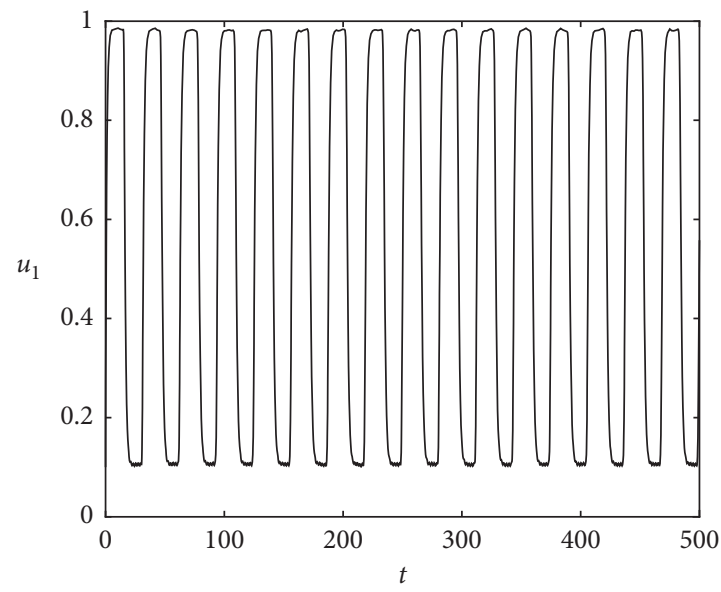

(d)

Figure 7: Time histories of system (20) with delay varying (a) $\tau=1.5$, (b) $\tau=1.6$, (c) $\tau=6$, and (d) $\tau=15$ for the fixed parameters $c_{1}=-3$, $c_{2}=-10, c_{3}=-3, c_{4}=-10, I_{1}=8$, and $I_{2}=8$.

delay increasing slightly, the top oscillation changes its position and enters into the down subthreshold oscillation, as shown in Figure 7(b) for time delay $\tau=1.6$. The neural system having multiple equilibria may employ time delay to realize Winner-Take-All functionality. Further, a periodic activity will be excited by increasing delay, as shown in Figure 7(c) for $\tau=6$. The oscillatory intensity of neural activity is stronger than the spiking illustrated in case 1 . The oscillation surrounds all top and down quiescent states. Finally, the periodic spiking transforms into a bursting-like behavior, as shown in Figure 7(d) for time delay $\tau=15$.

\section{Conclusion}

Complexity and dynamical analysis in neural systems play an important role in the application of optimization problem and associative memory. In this paper, we considered a delayed neural system with content/periodic external stimulations. The results show that content stimulations can induce and suppress a periodic activity. The neural system exhibits a periodic activity with delay increasing. Further, the system dynamics can be changed from quiescent state to periodic activity and then enter into the quiescent state with stimulation increasing. Additionally, in view of the ubiquitous rhythm in living organisms, we introduce the periodic stimulations with low frequency as the rhythm activity. The results show that the neural system subjected by periodic stimulations exhibits some interesting activities, such as the periodic spiking, subthreshold oscillation, and bursting-like ones. Further, with delay increasing slightly, the subthreshold oscillation can change its position from top to down. The neural system having multiple equilibria may employ time delay to realize Winner-TakeAll functionality.

\section{Data Availability}

All data, models, and code generated or used during the study are provided within the article.

\section{Conflicts of Interest}

The authors declared that they have no conflicts of interest regarding this work. 


\section{Acknowledgments}

This work was supported by the National Science Foundation of China (grant nos. 11672177 and 11672185).

\section{References}

[1] E. M. Izhikevich, "Neural excitability, spiking and bursting," International Journal of Bifurcation and Chaos, vol. 10, no. 6, pp. 1171-1266, 2000.

[2] M. Yao and R. Wang, "Neurodynamic analysis of merkel cellneurite complex transduction mechanism during tactile sensing," Cognitive Neurodynamics, vol. 13, no. 3, pp. 293-302, 2019.

[3] Z. Song, B. Zhen, and D. Hu, "Multiple bifurcations and coexistence in an inertial two-neuron system with multiple delays," Cognitive Neurodynamics, vol. 14, no. 3, pp. 359-374, 2020.

[4] X. Liao, K. W. Wong, and Z. Wu, "Bifurcation analysis on a two-neuron system with distributed delays," Physica D: Nonlinear Phenomena, vol. 149, no. 1-2, pp. 123-141, 2001.

[5] Z. Song, J. Xu, and B. Zhen, "Multitype activity coexistence in an inertial two-neuron system with multiple delays," International Journal of Bifurcation and Chaos, vol. 25, no. 13, p. 1530040, 2015.

[6] S. Sen, S. N. Daimi, K. Watanabe, K. Takahashi et al., "Switch or stay? automatic classification of internal mental states in bistable perception," Cognitive Neurodynamics, vol. 14, no. 1, pp. 95-113, 2020.

[7] H. R. Wilson and J. D. Cowan, "A mathematical theory of the functional dynamics of cortical and thalamic nervous tissue," Kybernetik, vol. 13, no. 2, pp. 55-80, 1973.

[8] A. Destexhe and T. J. Sejnowski, "The Wilson-Cowan model, 36 years later," Biological Cybernetics, vol. 101, no. 1, pp. 1-2, 2009.

[9] T. Ueta and G. Chen, "On synchronization and control of coupled Wilson-cowan neural oscillators," International Journal of Bifurcation and Chaos, vol. 13, no. 1, pp. 163-175, 2003.

[10] J. Harris and B. Ermentrout, "Bifurcations in the Wilson-cowan equations with nonsmooth firing rate," SIAM Journal on Applied Dynamical Systems, vol. 14, no. 1, pp. 43-72, 2015.

[11] R. M. Borisyuk and A. B. Kirillov, "Bifurcation analysis of a neural network model," Biological Cybernetics, vol. 66, no. 4, pp. 319-325, 1992.

[12] G. N. Borisyuk, R. M. Borisyuk, A. I. Khibnik, and D. Roose, "Dynamics and bifurcations of two coupled neural oscillators with different connection types," Bulletin of Mathematical Biology, vol. 57, no. 6, pp. 809-840, 1995.

[13] Y. Maruyama, Y. Kakimoto, and O. Araki, "Analysis of chaotic oscillations induced in two coupled Wilson-Cowan models," Biological Cybernetics, vol. 108, no. 3, pp. 355-363, 2014.

[14] L. H. A. Monteiro, A. P. Filho, J. G. Chaui-Berlinck, and J. R. C. Piqueira, "Oscillation death in a two-neuron network with delay in a self-connection," Journal of Biological Systems, vol. 15, no. 1, pp. 49-61, 2007.

[15] Z. Song and J. Xu, "Bursting near bautin bifurcation in a neural network with delay coupling," International Journal of Neural Systems, vol. 19, no. 5, pp. 359-373, 2009.

[16] Z. Song and J. Xu, "Codimension-two bursting analysis in the delayed neural system with external stimulations," Nonlinear Dynamics, vol. 67, no. 1, pp. 309-328, 2012.
[17] B. D. Noonburg and B. Pollina, "A periodically forced Wilson-Cowan system," SIAM Journal on Applied Dynamical Systems, vol. 63, no. 5, pp. 1585-1603, 2003.

[18] R. Decker and V. W. Noonburg, "A periodically forced Wilson-cowan system with multiple attractors," SIAM Journal on Mathematical Analysis, vol. 44, no. 2, pp. 887-905, 2012.

[19] Y. A. Kuznetsov, Elements of Applied Bifurcation Theory, Springer, New York, NY, USA, 1995.

[20] A. Mondal, R. K. Upadhyay, J. Ma, B. K. Yadav, S. K. Sharma, and A. Mondal, "Bifurcation analysis and diverse firing activities of a modified excitable neuron model," Cognitive Neurodynamics, vol. 13, no. 4, pp. 393-407, 2019.

[21] D. T. Kaplan, J. R. Clay, T. Manning, L. Glass et al., "Subthreshold dynamics in periodically stimulated squid giant axons," Physical Review Letters, vol. 76, no. 21, pp. 4074-4077, 1996.

[22] S. Chillemi, M. Barbi, and A. D. Garbo, "Dynamics of the neural discharge in snail neurons," Biosystems, vol. 40, no. 1-2, pp. 21-28, 1997.

[23] A. Szücs, R. C. Elson, M. I. Rabinovich, H. D. Abarbanel, and A. I. Selverston, "Nonlinear behavior of sinusoidally forced pyloric pacemaker neurons," Journal of Neurophysiology, vol. 85 , no. 4 , pp. $1623-1638,2001$. 